\title{
LA NUOVA FRONTIERA DEL FALSO: CLONARE LA BRAND EXPERIENCE
}

\author{
Claudio Sambri *
}

Nella Cina di oggi vi è un termine che viene sempre più spesso utilizzato per indicare la falsificazione, la contraffazione, l'imitazione servile ed al limite della legalità (o della illegalità), di un prodotto Questa parola è: shanzhai. Ovvero falso, "tarocco", contraffatto. È un termine utilizzato, particolarmente, ma non solo, in Cina per descrivere il mercato in costante crescita dei fake. Una zona grigia ed ambigua, nella quale trovano posto le più svariate imitazioni: dai Rolex alle borse "firmate" Louis Vuitton, dalle Nike ai pupazzi della Disney, per finire con i più sofisticati gadget elettronici, come gli iPhone o gli ormai mitici iPad della Apple. Copie conformi, spesso assai simili agli originali, ed altrettanto frequentemente disponibili a un prezzo ridotto, quando non addirittura assai significativamente ribassato. Ma che conservano standard di qualità assimilabili a quelli dei modelli originali.

I cosiddetti "pirati" cinesi, che si sono guadagnati, nel corso degli anni, per non dire dei decenni, una notevole fama per aver imitato (copiato) un gran numero di prodotti occidentali, sembrano aver deciso un cambio di strategia: la nuova frontiera del falso è il negozio clonato. Una blogger americana, trapiantata per lavoro nello Yunnan, provincia sud-occidentale della Cina, a metà luglio ha postato nel web le immagini di un falso Apple Store situato a Kunming ${ }^{1}$.

Questo store pone in vendita iPhone, MacBook e altri popolari prodotti della casa di Cupertino e ha, quale contenitore, un'incredibile rassomiglianza con gli originali Apple Store. Vi si possono vedere gli stessi tavoloni in legno con i prodotti a disposizione dei clienti; le stesse immagini

\footnotetext{
* Professore ordinario di marketing, Università degli Studi di Trieste (claudios@econ.units.it).

1. http://birdabroad.wordpress.com.
}

Mercati e Competitività n. 4, 2011 
pubblicitarie appese alle pareti; la medesima $t$-shirt di colore azzurro, che è poi la divisa dei commessi; la stessa scala a chiocciola che conduce alla sitting area al piano di sopra. Ma, a questo punto dell'indagine, si scopre che lo store non figura tra i rivenditori autorizzati della Apple, né tanto meno il gigante hi-tech ha mai aperto un proprio store nella provincia dello Yunnan. Il negozio Apple fake vende tuttavia merce originale, stando a quanto dichiara il titolare: presumibilmente (ipotizzano i cronisti che commentano il caso, assumendosi la responsabilità di quanto affermano) acquistata all'estero e contrabbandata in Cina per evadere le tasse. "Nessuna legge - puntualizza ad ogni buon conto il proprietario - impedisce di arredare il proprio negozio come si vuole".

Nelle settimane successive vengono alla luce numerose altre imitazioni, anche sull'onda di questa "tempesta" mediatica: falsi McDonald's, Starbucks di dubbia provenienza. Ma il vero, grande capolavoro della mise-enscene si trova ancora una volta a Kunming. È un finto negozio Ikea, assolutamente fasullo: ricostruito con cura nei minimi dettagli (cfr. Lee, 2011; Visetti, 2011): identico layout, colori giallo e blu, matitine gratuite, caffetteria e self service, area bimbi e servizio di montaggio per i mobili a pagamento. Unica differenza nel menu del ristorante: lo spezzatino di maiale con le uova al posto delle polpettine svedesi e del salmone marinato. Cambia anche il nome: il negozio si promuove come "11 Furniture", che in mandarino si pronuncia "Shi Yi Jia Ju", mentre Ikea è chiamata in modo molto simile: "Yi Jia Jia Ju"...

\section{Fiction economy: quando la merce fa spettacolo}

Si è di fronte ad un (apparente o reale?) cambio di passo nel mondo della contraffazione: si è cominciato vendendo prodotti falsi in negozi veri. E si finisce per vendere prodotti veri dentro negozi falsi. Dal contenuto al contenitore: in definitiva, l'intera brand experience diventa oggetto di clonazione $\mathrm{o}$, per essere più diretti e sinceri, di vera e propria "pirateria".

Da parte di taluni studiosi è soprattutto la differenza tra ciò che è reale e ciò che percepiamo come reale ad essere messa in discussione (Gilmore, Pine, 2007; trad. it., 2009, p. 49). Un tema di ben più vasta portata, che richiama l'attenzione sulla nuova prospettiva della fiction economy: un approccio, nel quale l'immaginario diventa componente strutturale del valore economico delle merci. In questo contesto il prodotto industriale diventa una sorta di "semilavorato", che - una volta uscito dalle linee di produzione - chiede di essere completato mediante un processo di valorizzazione simbolica. La catena del valore si estende quindi oltre i confini dell'impresa manifatturiera, integrando al proprio interno attività di design, di marketing, di comunicazione, fino alla vera e propria mise-en-scene realizzata al- 
l'interno del punto vendita: il processo produttivo finisce dunque per comprendere situazioni che in passato appartenevano all'economia dei servizi (Rullani, 2004), incorporando una dose crescente di "spettacolarità" e di "teatralizzazione".

D'altra parte, i prodotti della fiction economy non hanno soltanto una forte componente immateriale, ma diventano essi stessi forme indefinite o in-finite, supporti materiali per immagini, significati, narrazioni: merci-lifestyle (si pensi allo spremiagrumi di Philippe Starck), che chiedono di essere trasformate in discorso e racconto mediante la cooperazione di un ampio sistema di interpreti e di attori, secondo i classici paradigmi della Fiction economy (Carmagnola, 2006).

L'esito possibile di questi percorsi è duplice.

$\mathrm{Da}$ un lato, il progressivo coinvolgimento del consumatore finale (Thomke, von Hippel, 2002; Prahalad, Ramaswamy, 2004), incoraggiato a condividere con le aziende e con le marche tutte le dimensioni dell'esperienza, dalla ricerca delle informazioni alla configurazione del prodotto e del servizio, fino all'elaborazione dei significati e dei valori che danno senso al momento del consumo (Fabris, Rullani, 2007). Accogliendo queste suggestioni, anche la comunicazione d'impresa è chiamata ad accentuare l'impronta dialogica, trasformandosi da semplice veicolo di dati e informazioni in leva strategica del cambiamento: questo sviluppo implica infatti il trasferimento di quote crescenti di "sovranità" dalle imprese alle persone (Roberts, 2004).

Dall'altro lato, la riduzione a merce della stessa esperienza, con la scena che si sostituisce ai luoghi della vita (Augé, 1997; trad. it., 1999, p. 117). Oggi infatti le dinamiche della spettacolarizzazione si estendono al di fuori degli ambiti tradizionali, mettendo in crisi la stessa distinzione tra realtà e finzione e producendo un pericoloso fenomeno di inversione, per cui è la vita che imita il teatro, il reale che copia la finzione, e tutto assomiglia a un'immensa scenografia (op. cit., p. 65). Disneyland in questo senso non è che la metafora del mondo "derealizzato" in cui viviamo: un gioco di specchi, uno spettacolo che mette in scena se stesso, un continuo uscire da uno schermo per rientrare subito in un altro schermo (op. cit., pp. 17-25). Come suggerisce James W. Carey, oggi «la realtà è innanzitutto una risorsa scarsa» (Carey, 1992, p. 87).

Il caso del falso Apple Store di Kunming suggerisce a questo proposito tre ordini di considerazioni.

\subsection{Il prodotto: tra autenticità e finzione}

Il primo è riferito al prodotto, che oggi si muove sempre di più sul debole crinale che separa autenticità e finzione, secondo una logica di 
authentifactuation (Gilmore, Pine, cit., p. 81) ${ }^{2}$. In che senso gli iPhone e i MacBook venduti dal negozio fake possono essere considerati degli "originali"? Fino a che punto possiamo prestare ascolto a quanto dichiara - in apparente buona fede - il titolare?

La prima possibilità è che quelli venduti a Kunming siano dei veri Apple caduti, per così dire, "dal retro di un camion": che siano in altri termini dei prodotti autentici, in qualche modo finiti per qualche oscura ragione in un qualche canale alternativo a quello ufficiale. Senza scomodare troppo la fantasia, è anche possibile che vengano acquistati da un grosso dealer di Hong Kong, che li "rivende a un ingegnoso dettagliante di Kunming", abile nel mascherare il proprio punto vendita come se fosse un vero Apple Store. L'operazione è tecnicamente possibile, ma richiede uno sforzo organizzativo non indifferente, visto che il grosso centro dello Yunnan è il punto di riferimento per un' area popolata da oltre 7 milioni di abitanti.

La seconda possibilità è che siano dei prodotti autenticamente falsi: che siano cioè delle riproduzioni, delle imitazioni degli originali talmente ben fatte, da sembrare addirittura autentiche. In realtà, si tratterebbe di pezzi assemblati da un anonimo terzista, che utilizza le tecniche di reverse engineering con il preciso intento di riprodurre una copia perfetta dell'originale, mettendo assieme materiali e componenti - monitor, hard disk, tastiere, processori - ampiamente disponibili sul mercato dei pezzi di ricambio. È un'operazione che fa pensare ai quadri di Bansky o di Myatt: copie di Van Gogh, Picasso e Giacometti, che hanno catturato l'attenzione dei critici d'arte e dei collezionisti per la straordinaria somiglianza con gli originali, al punto da trovare spazio al MOMA o alla Tate Modern (op. cit., p. 80). Una logica non troppo diversa da quella dei prodotti "fintentici" (op. cit., p. 169), cioè dichiaratamente falsi, tipo gli orologi proposti da marche come Buluga, Courtier o Modova, che suonano familiari, pur affermando esplicitamente di non essere Bulova, Cartier o Movado. Con la differenza che il negozio di Kunming spaccia per autentiche quelle che sono semplici copie realizzate da abili falsari.

L'ultima possibilità è che siano prodotti falsamente autentici: si tratterebbe in questo caso di eccedenze di produzione realizzate dalla Foxconn (la ditta cinese che li assembla in nome e per conto dell'americana Apple), che andrebbero ad alimentare una sorta di cono d'ombra, ossia un canale distributivo "grigio", parallelo ma non alternativo a quello ufficiale, per quanto sottratto al controllo diretto dell'azienda di Steve Jobs. In questo caso i prodotti sarebbero in tutto e per tutto identici, quanto a funzionalità, caratteristiche tecniche e prestazioni, agli omologhi dispositivi venduti nei negozi autorizzati. Pur essendo marchiati con il famoso simbolo della mela

2. Authenticfactuation: neologismo, - traducibile in italiano con "autentirealtà" - lanciato dalla rivista Fortune nel 2002 e riproposto da Gilmore e Pine (2007). 
morsicata, non sarebbero tuttavia da considerare come dei "veri" Apple nel senso stretto del termine, in quanto verrebbero a mancare - in questo, come nel caso precedente - $\mathrm{i}$ codici di autenticazione che consentono all' $u$ tente di connettersi ad AppleCare e di registrarsi al servizio di assistenza. Non sarebbero in altri termini prodotti "mappati", "taggati", riconosciuti ufficialmente dalla casa madre: mancando ogni forma di tracciabilità, verrebbe meno il criterio fondamentale per poter stabilire l'autenticità del computer, del tablet o del telefonino appena acquistato.

Questo punto sembra particolarmente interessante: non sono le caratteristiche intrinseche del prodotto (materiali utilizzati, qualità dei componenti e delle finiture, funzionalità, prestazioni) a farsi garanti della sua autenticità, ma unicamente la disponibilità di un codice di registrazione e di accesso, che colleghi la singola device a un più ampio sistema di connessioni e di significati. Non soltanto servizi di assistenza: ma anche narrazioni, miti di fondazione, simboli e valori, futuri possibili. Un fenomeno che coinvolge in misura crescente molteplici settori.

DOC, DOP, IGP, STG: già oggi nell'ambito dell'industria alimentare la tracciabilità e le certificazioni di origine geografica diventano per certi aspetti quasi più importanti dei prodotti. Non si compra più un prosciutto, ma un "Parma" o un "San Daniele". Possiamo dunque immaginare un domani in cui non acquisteremo più prodotti, ma patenti, certificati di qualità e di garanzia, codici d'accesso, licenze d'uso. Come Chris Anderson (2009) suggerisce tra le righe, il prodotto tecnologico potrà anche esser regalato, ovvero concesso in prova per un periodo di tempo limitato: solo l'acquisto di una specifica "chiave" lo renderà perfettamente funzionante. Un po' come accade, già oggi, per i cellulari.

In definitiva, "la vita stessa richiederà un qualche marchio di autenticità". Una prospettiva, che fa pensare agli inquietanti scenari delineati nel film di fantascienza Gattaca (1997): un complesso sistema di riconoscimento consente soltanto agli individui concepiti in provetta di accedere ai ruoli sociali e alle professioni più importanti, lasciandone del tutto esclusi quanti - concepiti in modo naturale - non possono vantare il "pedigree" di un corredo geneticamente perfetto.

Questo modo di risolvere il problema (è autentico solo ciò che dispone di un marchio di garanzia che lo certifichi come tale) ha in sé qualcosa di paradossale. Parafrasando Watzlawick (1967), non si può pretendere che un'esperienza sia autentica, se ciò viene richiesto per decreto: in maniera analoga, non si può restituire alle persone il sapore dell'esperienza autentica e vissuta, se questa è costruita a tavolino, definita in maniera artificiale. In sostanza, la continua necessità di dichiarare che qualcosa è "autentico", certificato, originale, fa immediatamente dubitare i consumatori della sua autenticità (Gilmore, Pine, cit., p. 82). 


\subsection{Il punto vendita: se il luogo viene prima dell'oggetto}

Il secondo ordine di considerazioni è riferito al punto vendita. Paradossalmente, il caso cinese dei falsi Apple Store non fa che confermare quanto viene sottolineato da anni nell'ambito del marketing: il contenitore è diventato più importante del contenuto. In altri termini, il luogo viene prima dell'oggetto. Oggi infatti l'individuo tende a spostare la sua scelta dal prodotto al negozio: di conseguenza, anche il processo decisionale riferito al bene da acquistare è fortemente condizionato dal punto vendita selezionato (Castaldo, Botti, 1999). Il processo d'acquisto è infatti motivato da un'ampia gamma di giustificazioni, alcune direttamente legate alla necessità di acquistare un bene determinato, altre che fanno riferimento alla dimensione ludica, edonistica e ricreativa dello shopping (cfr. Hirschmann, Holbrook, 1982). Per essere adeguatamente soddisfatti, questi moventi richiedono una rilettura del punto vendita in termini di comunicazione, al fine di poter offrire un'esperienza d'acquisto stimolante e coinvolgente (Sambri, Pegan, 2003; Qualizza, 2006).

Si pensi al caso di Build-a-Bear, azienda che ha saputo rinnovare il business un po' stantio dell' orsetto di peluche, lavorando sulle caratteristiche del punto vendita e sulla relazione con il consumatore finale: il risultato è una sorta di workshop, un negozio-laboratorio, nel quale il cliente ha l'opportunità di creare un prodotto personalizzato, interagendo con il personale di vendita e con le innumerevoli sollecitazioni proposte. Ma si pensi anche al marketing museale: l'avveniristica struttura al titanio che avvolge il Guggenheim di Bilbao, progettato da Frank Gehry, è di per sé un'opera d'arte che attrae milioni di visitatori da tutto il mondo, contribuendo in maniera decisiva al rilancio come meta turistica di quella che è stata per lungo tempo una città a prevalente vocazione industriale.

Applichiamo queste considerazioni al caso di Apple: tutti i suoi prodotti sono - in qualche misura - dei fake, in quanto realizzati materialmente da un terzista cinese (Foxconn) e non direttamente dall'azienda di Cupertino. Ciò che resta di autentico nella marca Apple è dunque rappresentato oltre che dal design concept e dalle attività di ricerca e sviluppo - proprio dal punto vendita, cioè dal principale touch point attraverso il quale l'azienda dialoga con i suoi utenti. Potremmo anzi dire che il vero prodotto di Apple è, in definitiva, lo stesso cliente, che vive nello store un'esperienza di trasformazione (Pine, Gilmore, 1999), avvicinandosi a un diverso modo di intendere il proprio rapporto con le nuove tecnologie.

Sempre muovendoci sul filo di questi ragionamenti, non possiamo non accennare al tema del packaging, che oggi si arricchisce di nuove funzioni, in aggiunta a quelle tradizionali di protezione e conservazione del contenuto (cfr. Ferraresi, 1999). Gli aspetti di comunicazione vengono dunque privilegiati: forma, colore, lettering, disposizione delle scritte sull'involucro 
devono non solo offrire informazioni su quanto sta all'interno, ma anticipare in chiave metaforica una possibile esperienza del prodotto e del brand. In taluni casi, come quello della Coca-Cola, del Campari Soda, della Moka Bialetti, della Mini BMW, del Maggiolino Volkswagen, la forma è talmente evocativa della marca, da rappresentare per il consumatore la marca stessa, dando luogo a un processo di "iconizzazione della forma", che ha effetti immediati e duraturi sulla brand equity e sulla brand awereness. In definitiva, la Coca-Cola è la sua bottiglia.

\subsection{La marca come universo simbolico e valoriale}

Da ultimo, va considerato il ruolo svolto dalla marca, che oggi assume un carattere di riferimento permanente, comunicativo e valoriale, capace di superare l'erosione dei cicli di vita dei singoli prodotti, la saturazione dei beni di consumo tradizionali e la progressiva dematerializzazione degli oggetti (Semprini, Musso, 2001; Pratesi, Mattia, 2006).

Nel corso degli anni le imprese hanno infatti spostato l'accento sulle competenze soft (know-how, professionalità dei dipendenti, qualità del servizio, cultura organizzativa, innovazione), e in particolare sulla capacità di creare valore attraverso gli aspetti simbolici. Il risultato è uno slittamento dei termini e dei significati, per cui non parliamo più di marchio (semplice "etichetta" applicata ad un prodotto), ma di marca, intesa come dispositivo comunicazionale, progetto di senso che dà vita a un mondo possibile, ad un vero e proprio immaginario (Semprini, 1996).

In sostanza, si passa dal mercato del prodotto al mercato della marca: se nel mercato tradizionale ciò che veniva scambiato erano esclusivamente prodotti, ovvero oggetti fisici, con le loro funzionalità e con le loro prestazioni, nel nuovo mercato ciò che produttori e consumatori si scambiano sono essenzialmente discorsi e gli stessi prodotti "esistono", soltanto nella misura in cui trovano collocazione nell'universo simbolico e valoriale evocato dalle marche (cfr. Musso, 2011).

Il caso del falso Apple Store conferma paradossalmente la validità di questi assunti: il brand è molto più di un semplice logo posizionato su una borsa da viaggio o di una mela disegnata sulla case di un computer di tendenza. Non si copiano più i prodotti con le loro etichette, ma le esperienze e i discorsi di marca.

\section{Discorsi di marca e modelli di business: due diversi ap- procci}

Parlando di realtà leader nel mondo delle tecnologie dell'informazione e della comunicazione, vale tuttavia la pena sottolineare la differenza tra 
due diversi approcci al tema della marca: da un lato, il sistema chiuso realizzato da Apple, dall'altro lato, il sistema aperto messo a punto da Android con il fondamentale supporto di Google.

Nel primo caso, l'ossessione quasi maniacale per la coerenza della comunicazione porta alla creazione di un vero e proprio brandscape, un paesaggio di marca definito con rigore anche nei minimi dettagli, che si propone agli occhi del consumatore come un mondo globale di riferimento, dotato di regole, valori e comportamenti autonomi: una situazione indubbiamente suggestiva, che si caratterizza tuttavia per un eccesso di forza e di coerenza, spesso percepito come una forma di "totalitarismo estetico", pregiudicando il coinvolgimento attivo dell'interlocutore (cfr. Schmitt, Simonson, 1997, p. 283), che infatti Apple non ha mai esplicitamente incoraggiato, in quanto pretende un controllo completo sui suoi prodotti. Il modello di business non è infatti legato esclusivamente alla vendita di device tecnologiche, come iPod, MacBook e iPhone, ma anche alle vendite complementari di software, contenuti e periferiche: ad esempio, il redditizio mercato dei brani musicali acquistabili tramite iTunes. Consentire agli utenti di modificare liberamente i prodotti e il relativo software equivarrebbe a perdere il controllo sulle proprie piattaforme, magari ponendo le basi per l'apparizione di altri concorrenti (Tapscott, Williams, 2007, pp. 148-54). L'unica "libertà" concessa di fatto ai propri interlocutori è dunque quella di sviluppare la logica del ready made, misurandosi nell'impegnativa sfida di ricreare copie in tutto e per tutto identiche alle forme degli originali. Non a caso, pullulano nel web i siti che spiegano come trasformare il proprio $\mathrm{Pc}$ in un fake somigliante, quanto più possibile, a un Mac: paradossalmente, è lo spirito del "sistema chiuso" a incoraggiare la copia e la contraffazione. Non solo del prodotto: anche del punto vendita e della brand experience.

Android al contrario si fa vanto della mancanza di controllo (cfr. Priolo, 2011): mette gratuitamente a disposizione il codice sorgente del suo sistema operativo open source, non revisiona le app prima che siano immesse nel mercato e lascia ai produttori ampio margine per modificare le funzioni e il look del sistema operativo. Si realizza in questo modo un ecosistema, al quale contribuiscono tutte le aziende che equipaggiano i propri prodotti con Android: da Samsung ad LG, da HTC a Motorola, incoraggiate a realizzare migliaia di dispositivi - tablet e cellulari - ogni volta differenti, in coerenza con le cangianti attese dei propri clienti. Nessuno di questi dispositivi ha lo stesso successo dell'iPad o dell'iPhone, ma - messi tutti assieme - raggiungono una base di utenti molto più ampia e dunque più attraente per gli sviluppatori di applicazioni. Anche la community degli utenti è costantemente sollecitata a realizzare forme di peer production e di miglioramento delle applicazioni: nel mondo del software libero, la distinzione tra originale e fake viene destituita di ogni significato. 
In coerenza con questo modello di business, che si avvicina all'ideale dell'impresa eterarchica (Valdani, 2000), Android (alias Google) ha colto l'opportunità di sviluppare un "template di marca" dinamico, coerente nei valori di fondo, ma adattabile a contesti differenti, lontano in ogni caso dall'astratto rigore formale dei manuali di corporate identity utilizzati dalle aziende nel passato (Pini, Gonella, 2002 p. 292).

\section{Conclusioni}

Emergono, da questo confronto, due diversi modi di definire l'autenticità di un'esperienza, differenti ma complementari.

Nel primo caso (Apple), è autentico tutto ciò che è unico, esclusivo, non convenzionale e non banale, e pertanto disponibile soltanto in edizione limitata: in definitiva, è autentico tutto ciò che rappresenta la porta d'accesso a un più nobile scopo, in coerenza con le caratteristiche di "marca-missione" che l'azienda si è data.

Nel secondo caso (Android), un'esperienza appare tanto più autentica, quanto più i consumatori hanno contribuito a generarla, in coerenza con il modello di "marca-euforia", conviviale e partecipativa, che l'azienda si è impegnata a sviluppare. Autentico è dunque tutto ciò che è legato all'assenza di un copione: tutto ciò che è poligonale e si discosta da una forma predefinita. Tutto ciò che spiazza, sorprende, seduce. Liberando dall'obbligo della coerenza fine a se stessa.

\section{Riferimenti bibliografici}

Anderson Ch. (2009). Free. The Past and Future of a Radical Price. New York, Hyperion; trad. it. di I.Katerinov, Gratis. Milano:Rizzoli, 2009.

Augè M. (1997). L'impossible voyage. Le tourisme et ses images. Paris, Payot \& Rivages; trad. it. di A. Salsano, Disneyland e altri nonluoghi. Torino: Bollati Boringhieri, 1999.

Carey J.W. (1992). Communications as Culture: Essays on Media and Society. New York: Routledge.

Carmagnola F. (2006). Il consumo delle immagini. Estetica e beni simbolici nella fiction economy. Milano: Bruno Mondadori.

Castaldo S., Botti S. (1999). Lo shopping emozionale. Una ricerca esplorativa sul ruolo del punto vendita. Economia\&Management: 1, 17-37.

Fabris G.P., Rullani E. (2007). Il consumatore creativo. Cento e uno modi di fare il mondo a propria immagine e somiglianza. Economia e politica industriale: 7-24.

Ferraresi M. (1999). Il packaging. Oggetto e comunicazione. Milano: FrancoAngeli.

Gilmore J.H., Pine B.J. (2007). Authenticity. Boston, Harvard Business School Press; trad. it. di V. Prandoni, Autenticità. Ciò che i consumatori vogliono davvero. Milano: FrancoAngeli, 2009. 


\section{Sambri}

Hirschmann E., Holbrook M. (1982). Hedonic Consumption: Emerging Concepts, Methods and propositions. Journal of Marketing: 46, 92-101.

Lee M. (2011). Chinese retailers hijack the Ikea experience. Reuters.com, 1 agosto.

Musso, P. (a cura di) (2011). Brand Reloading. Nuove Strategie per comunicare, rappresentare e raccontare la marca. Milano: FrancoAngeli.

Pine J., Gilmore J.H. (1999). The Experience Economy. Work Is Theatre \& Every Business a Stage. Boston: Harvard Business School Press; trad. it. di A. ScottMonkhouse. L'economia delle esperienze. Milano: Etas, 2000.

Pini M., Gonella F. (2002). Il ruolo della marca nell' economia digitale: verso un nuovo modello gestionale? Micro \& Macro Marketing: 281-297.

Prahalad C.K., Ramaswamy, V. (2004). The future of competition. Co-creating unique value with customers. Boston, Harvard Business School Press; trad. it. di F. Guaraldo e R. Ricca. Il futuro della competizione. Co-creare valore eccezionale con $i$ clienti. Milano: Il sole 24 ore, 2004.

Pratesi C.A., Mattia G. (2006). Branding. Strategia, organizzazione, comunicazione e ricerche per la marca. Milano: McGraw-Hill.

Priolo S. (2011). Il cuore Android di Mr. Rubin. Wired, n. 28, giugno, pp. 80-88.

Qualizza G. (2006). Oltre lo shopping. I nuovi luoghi del consumo: percorsi, esplorazioni, progetti. Trieste: Edizioni Goliardiche.

Roberts K. (2004). Lovemarks. The Future beyond Brands. New York: PowerHouse Books, 2004; trad. it. di G. Russo, Lovemarks. Il futuro oltre i brand. Milano: Mondadori, 2005.

Rullani E. (2004). La fabbrica dell'immateriale. Roma: Carocci.

Sambri C., Pegan G. (2003). Strategie di comunicazione di marketing. Il ruolo del punto vendita. Trieste: Edizioni Goliardiche.

Schmitt B., Simonson A. (1997). Marketing aesthetics. The strategic management of brands. identity, and image. New York: The Free Press.

Semprini A. (1996). La marca. Dal prodotto al mercato, dal mercato alla società. Milano: Lupetti.

Semprini A., Musso P. (2001). Dare un senso alla marca. In Lombardi M. (a cura di). Il dolce tuono. Marca e pubblicità nel terzo millennio. Milano: FrancoAngeli, pp. 43-66.

Tapscott D., Williams, A.D. (2006). Wikinomics. How Mass Collaboration Changes Everything, New York, Portfolio; trad. it. M. Vegetti. Wikinomics. La collaborazione di mass che sta cambiando il mondo. Milano: Etas, 2007.

Thomke S., von Hippel E. (2002). Customers as Innovators. Harvard Business Review, aprile, pp. 74-81.

Valdani E. (2000). L'impresa pro-attiva: co-evolvere e competere nell'era dell'immaginazione. Milano: McGraw-Hill.

Visetti G. (2011). In Cina negozi Ikea clonati. La nuova frontiera del falso. Repubblica, 2 agosto, pp. 1 e 34.

Watzlawick P. \& alii (1967). Pragmatic of human communication. A study of intercultural patterns, pathologies and paradoxes. New York: Norton \& Co; trad. it. M. Ferretti. Pragmatica della Comunicazione umana. Roma: Astrolabio, 1971. 